PROCEEDINGS OF THE

AMERICAN MATHEMATICAL SOCIETY

Volume 135, Number 8, August 2007, Pages 2381-2389

S 0002-9939(07)08769-2

Article electronically published on March 29, 2007

\title{
ACTIONS OF POINTED HOPF ALGEBRAS WITH REDUCED PI INVARIANTS
}

\author{
PIOTR GRZESZCZUK AND MAŁGORZATA HRYNIEWICKA
}

(Communicated by Martin Lorenz)

\begin{abstract}
Let $R$ be an $H$-module algebra, where $H$ is a pointed Hopf algebra acting on $R$ finitely of dimension $N$. Suppose that $L^{H} \neq 0$ for every nonzero $H$-stable left ideal of $R$. It is proved that if $R^{H}$ satisfies a polynomial identity of degree $d$, then $R$ satisfies a polynomial identity of degree $d N$ provided at least one of the following additional conditions is fulfilled:

(1) $R$ is semiprime and $R^{H}$ is almost central in $R$,

(2) $R$ is reduced.

If we also assume that $R^{H}$ is central, then $R$ satisfies the standard polynomial identity of degree $2[\sqrt{N}]$, where $[\sqrt{N}]$ is the greatest integer in $\sqrt{N}$.
\end{abstract}

\section{INTRODUCTION}

This paper is motivated by the following general question: if $H$ is a finitedimensional Hopf algebra over the field $K$, and $R$ is a left $H$-module algebra such that the algebra of invariants $R^{H}$ satisfies a polynomial identity, must $R$ also satisfy a polynomial identity? The answer to this question is positive in many concrete situations, e.g.,

(1) when $H=K[G]$, where $G$ is a finite group, and either $|G|^{-1} \in K$ or $R$ is reduced (see [K1] and [K2]);

(2) when $H=K[G]^{*}$ (see $[\mathrm{BC}]$ and $[\mathrm{BaZ}]$ );

(3) when $H=u(L)$, where $L$ is a finite-dimensional restricted Lie algebra of derivations of a prime ring $R$ with char $R=p>0$ such that $R^{H}$ is semiprime and the elements inducing the $X$-inner part of $L$ generate a quasi-Frobenius algebra (see [K3]);

(4) when $H$ is such that for every $H$-module algebra $R$ such that $R^{H}$ is nilpotent, also $R$ is nilpotent (see $\mathrm{BaL}$ );

(5) when $H$ is pointed and $R$ contains an element $\gamma$ such that $t \cdot \gamma=1$, for some $0 \neq t \in \int_{H}^{l}$, the space of left integrals of $H$ (see $[\mathrm{BeT}$ ).

However for actions of finite groups, where $|G| R=0$, it is known that the answer can be negative. In an example of Bergman, there is an action of a group $G$ of order $p^{2}$ (where $p$ is the characteristic of $K$ ) on the algebra $R=M_{2}(K[x, y])$ of $2 \times 2$ matrices over a free algebra $K[x, y]$ such that $R^{G}$ is commutative. Recall that in

Received by the editors January 8, 2006 and, in revised form, April 25, 2006.

2000 Mathematics Subject Classification. Primary 16R20, 16S40, 16W30.

The first author was supported by Polish KBN grant No. 1 P03A 03227.

(C)2007 American Mathematical Society 
this example, $G$ is generated by the inner automorphisms induced by

$$
\left(\begin{array}{ll}
1 & x \\
0 & 1
\end{array}\right) \text { and }\left(\begin{array}{ll}
1 & y \\
0 & 1
\end{array}\right)
$$

Then $R$ is a prime ring where every nonzero $G$-stable left (right) ideal of $R$ contains nontrivial invariants. This shows that the assumption that every nonzero $H$-stable left ideal of $R$ contains nontrivial invariants is not sufficient to obtain a positive answer to the above question. Notice that in the above example $R^{H}$ contains nilpotent elements. The main goal of this paper is to present a condition, which guarantees, for a semiprime algebra $R$, that if $R^{H}$ satisfies a PI, then $R$ also satisfies a PI. We will show that if $H$ is pointed and every nonzero $H$-stable left ideal contains a nontrivial central invariant, then $R^{H}$ satisfying a PI implies that $R$ satisfies a PI. This extends a situation considered in [C] and [CW], where actions of Hopf algebras with central invariants are studied. The PI condition for prime rings with central rings of invariants under the action of $p$-nilpotent groups, nilpotent Lie algebras and Lie superalgebras was also considered in $\overline{B C F}$ and $[\mathrm{BG}$. In the second main result, we show that if $R$ has no nonzero nilpotent elements, then the assumption that nonzero $H$-stable left ideals contain nontrivial invariants is sufficient for lifting the PI property from $R^{H}$ to $R$. Note that the most typical nontrivial examples of pointed Hopf algebras, which are neither group algebras nor universal enveloping algebras, are given by Lusztig's finite-dimensional Hopf algebras $\mathfrak{u}_{q}(\mathfrak{g})$ arising from quantized enveloping algebras at roots of unity for semisimple Lie algebras $\mathfrak{g}$ (see AS).

Throughout the paper $K$ will be a field, $H$ a pointed Hopf algebra over $K$, and $R$ an algebra over $K$. We let $\Delta: H \rightarrow H \otimes H$ be the comultiplication of $H, \epsilon: H \rightarrow K$ is the counit of $H$, and $S: H \rightarrow H$ the antipode of $H$. We say that $R$ is a left $H$-module algebra if $R$ is a left $H$-module such that $h \cdot a b=\sum\left(h_{1} \cdot a\right)\left(h_{2} \cdot b\right)$ and $h \cdot 1_{R}=\epsilon(h) 1_{R}$, where $h \in H, \Delta(h)=\sum h_{1} \otimes h_{2}, a, b \in R$. If $A$ is a subset of $R$ such that $h \cdot A \subseteq A$, for all $h \in H$, then we say that $A$ is $H$-stable. When $R$ is a left $H$-module algebra one can consider the smash product $R \# H$. As a vector space $R \# H$ is $R \otimes H$. The elements of $R \# H$ can be written as finite sums $\sum a_{h} h$, where $h \in H$ and $a_{h} \in R$. Then the multiplication in $R \# H$ is determined by the formula $(a h)(b l)=\sum a\left(h_{1} \cdot b\right) h_{2} l$, for all $a, b \in R$ and $h, l \in H$. The ring of invariants $R^{H}$ is defined as $\{r \in R \mid h \cdot r=\epsilon(h) r$, for all $h \in H\}$.

If $R$ is a left $H$-module algebra, then $R$ becomes a left $R \# H$-module using the left action $(a h) \cdot r=a(h \cdot r)$, where $a, r \in R$ and $h \in H$. Then the commuting ring $\operatorname{End}_{R \# H}(R)$ is isomorphic to $R^{H}$ and the submodules of $R$ over $R \# H$ are precisely left $H$-stable ideals of $R$.

If $M$ is a left $H$-module, then there is a homomorphism $\pi: H \rightarrow \operatorname{End}_{K}(M)$ defined by $\pi(h)(m)=h m$, for all $h \in H$ and $m \in M$. If $\operatorname{dim}_{K} \pi(H)=N<\infty$, then we say that $H$ acts finitely of dimension $N$. Clearly $\operatorname{dim}_{K} \pi(H) \leq \operatorname{dim}_{K} H$, so if $H$ is finite dimensional, then $H$ acts finitely on each $H$-module.

If $R$ is semiprime, we let $Q=Q(R)$ denote the symmetric Martindale quotient ring. Its center, known as the extended centroid of $R$, we denote by $C$. The following properties of $Q$, when $R$ is acted on by a Hopf algebra, are proved in Propositions 1, 2 and 5 of [GH]. 
Lemma 1. Let $R$ be a semiprime $H$-module algebra such that the $H$-action on $R$ extends to an $H$-action on $Q$ and any nonzero $H$-stable ideal of $R$ contains nontrivial invariants. Then

(1) the ring $C^{H}=C \cap Q^{H}$ is von Neumann regular and selfinjective.

(2) For any nonempty subset $X$ of $Q$ there exists an idempotent $\widehat{e}_{X} \in C^{H}$ such that $\operatorname{ann}_{C^{H}}(X)=\left(1-\widehat{e}_{X}\right) C^{H}$. If $X$ is an injective $C^{H}$-submodule of $Q$, then there exists $x \in X$ such that $\operatorname{ann}_{C^{H}}(X)=\operatorname{ann}_{C^{H}}(x)=\left(1-\widehat{e}_{x}\right) C^{H}$.

(3) If $L \subseteq Q$ is an $H$-stable subalgebra of $Q$ which is injective as a $C^{H}$-module, then $L^{H}$ is also injective as a $C^{H}$-module.

(4) If a nonempty subset $S \subseteq C^{H} \backslash\{0\}$ is closed under a multiplication, then the localization $Q_{S}$ of $Q$ at $S$ is semiprime and $Z\left(Q_{S}\right)=C_{S}$.

(5) If $H$ acts finitely on $Q$ and $S=C^{H} \backslash M$, where $M$ is a maximal ideal of $C^{H}$, then the $H$-action on $Q$ extends to an $H$-action on $Q_{S}$ and $\left(Q^{H}\right)_{S}=$ $\left(Q_{S}\right)^{H}$, where $\left(C^{H}\right)_{S}=\left(C_{S}\right)^{H}=C_{S} \cap\left(Q_{S}\right)^{H}$ is a field contained in the center of $Q_{S}$.

It is also known ( $[\mathrm{GH}$, Proposition 2]) that under the assumptions of Lemma 1 the $\operatorname{ring} Q$ is nonsingular and injective as a $C^{H}$-module. This immediately implies that if $\varphi: M \rightarrow N$ is an onto $C^{H}$-module map, where $0 \neq N \subseteq Q$ and $M$ is injective, then $N$ is also an injective $C^{H}$-module. In particular, any principal left ideal $Q q$ of $Q$ is nonsingular and injective as a $C^{H}$-module. Hence each finitely generated left ideal of $Q$ (finitely generated as a left $Q$-module) is also injective over $C^{H}$.

An important role will be played by the following result of Bergen, Cohen and Fischman on irreducible actions of Hopf algebras (see $[\mathrm{BCF}$, Theorem 2.2).

Theorem 2. Let $A$ be a left $H$-module algebra such that $A \# H$ acts irreducibly on $A, A$ has a finite left Goldie rank, and $H$ acts finitely of dimension $N$ on $A$. Then $\left[A: A^{H}\right]_{r} \leq N$, where $\left[A: A^{H}\right]_{r}$ is the dimension of $A$ as a right vector space over the division ring $A^{H}$.

\section{MAin Results}

Throughout this section $H$ will be a pointed Hopf algebra over a field $K$. Recall that a ring $R$ is said to be reduced if it does not contain nonzero nilpotent elements. It is well known that if $r_{1}, r_{2}, \ldots, r_{n}$ are elements of a reduced ring $R$ such that $r_{1} r_{2} \ldots r_{n}=0$, then $r_{f(1)} r_{f(2)} \ldots r_{f(n)}=0$ for any bijection $f:\{1,2, \ldots, n\} \rightarrow$ $\{1,2, \ldots, n\}$.

If $R$ is a left $H$-module algebra with center $Z(R)$, we say that the ring of invariants is almost central in $R$ if $L^{H} \cap Z(R) \neq 0$ for every nonzero $H$-stable left ideal $L$ of $R$. Notice that if $R$ is semiprime and $R^{H}$ is almost central in $R$, then $R^{H}$ is reduced. Indeed, suppose there exists $0 \neq a \in R^{H}$ such that $a^{2}=0$. The left ideal $R a$ is $H$-stable, so one can find a nonzero element $r a \in(R a)^{H} \cap Z(R)$. Then ara $=a(r a)=(r a) a=0$ and thus $(r a)^{2}=0$, which is impossible since $Z(R)$ is reduced.

Our first main goal is to prove the following.

Theorem 3. Let $R$ be a semiprime $K$-algebra with center $Z$ and suppose $R$ is a left $H$-module algebra, where $H$ is a pointed Hopf algebra acting on $R$ finitely of

dimension $N$. If the subalgebra of invariants $R^{H}$ is almost central in $R$, and $R^{H}$ 
satisfies a polynomial identity of degree $d$, then $R$ satisfies the standard polynomial identity of degree $d N$. If in addition $R^{H} \subseteq Z$, then $R$ satisfies the standard polynomial identity of degree $2[\sqrt{N}]$, where $[\sqrt{N}]$ is the greatest integer in $\sqrt{N}$.

Our next result concerns the situation when the algebra $R$ is reduced.

Theorem 4. Let $R$ be a reduced $H$-module $K$-algebra, where $H$ is a pointed Hopf algebra acting on $R$ finitely of dimension $N$. Suppose that $L^{H} \neq 0$ for every nonzero $H$-stable left ideal $L$ of $R$. If $R^{H}$ satisfies a polynomial identity of degree $d$, then $R$ satisfies the standard polynomial identity of degree $d N$.

The proofs require some preparation. Recall that a module $M$ is called uniform if the intersection of any two nonzero submodules is nonzero. We start with the following general observation.

Lemma 5. Let $M$ be an irreducible (uniform) left $R \# H$-module and suppose that $H$ acts finitely on $M$. Then $M$ has finite length (finite Goldie rank) as a left $R$-module.

Proof. Let $M$ be an arbitrary (not necessarily irreducible) left $R \# H$-module. Let $\pi: H \rightarrow \operatorname{End}_{K}(M)$ be a homomorphism of algebras induced by the action of $H$ on $M$. By using the Taft-Wilson Theorem (see [M1, Theorem 5.4.1]) we can decompose $H$ as a finite union $\bigcup_{i=1}^{N} H_{i}$ of an increasing chain of subspaces $\left\{H_{i}\right\}$ such that

(i) $\pi\left(H_{i}\right)=\pi\left(H_{i-1}\right)+K \cdot \pi\left(h_{i}\right)$, where $h_{1}=1_{H}$ and $h_{i} \in H$ for $2 \leq i \leq N$,

(ii) $\Delta\left(h_{i}\right) \in \sigma \otimes h_{i}+h_{i} \otimes \tau+H_{i-1} \otimes H_{i-1}$, where $\sigma, \tau \in G$ and $2 \leq i \leq N$.

Moreover, we can assume in (ii) that if $h_{i} \neq \tau$ (that is, if $h_{i}$ is not a group-like element), then $\tau \in H_{i-1}$.

If $A$ is an $R$-submodule of $M$ and $j \geq 1$, let

$$
A_{(j)}=\left\{m \in M \mid h_{1} m, \ldots, h_{j} m \in A\right\} .
$$

If $h_{i} \in H$ satisfies (ii), then

$$
h_{i}(r m)=\sigma(r) h_{i} m+\left(h_{i} \cdot r\right) \tau m+\sum\left(h_{i 1} \cdot r\right) h_{i 2} m,
$$

where $r \in R, m \in M$ and $h_{i 1}, h_{i 2} \in H_{i-1}$. Thus an easy induction argument shows that $A_{(j)}$ is also an $R$-submodule of $M$. Since $\left\{\pi\left(h_{1}\right), \ldots, \pi\left(h_{N}\right)\right\}$ is a $K$-basis of $\pi(H)$, we obtain immediately that $h A_{(N)} \subseteq A_{(N)}$, for all $h \in H$; thus $A_{(N)}$ is an $R \# H$-submodule. In fact $A_{(N)}$ is the largest $R \# H$-submodule contained in $A$. Now if $\left\{A_{\alpha}\right\}$ is a chain of $R$-submodules of $M$, each of which contains no nonzero $R \# H$-submodule, then $\bigcup A_{\alpha}$ also contains no nonzero $R \# H$-submodule. Indeed, if $B \subseteq \bigcup A_{\alpha}$ is a nonzero $R \# H$-submodule and $0 \neq b \in B$, then $\left\{h_{1} b, \ldots, h_{N} b\right\} \subseteq A_{\alpha_{0}}$ for some $\alpha_{0}$. Therefore $(R \# H) b \subseteq A_{\alpha_{0}}$, and so $A_{\alpha_{0}}$ contains a nonzero $R \# H$ submodule, a contradiction. Consequently, by Zorn's Lemma, there exists an $R$ submodule $\widehat{A}$ of $M$ which is maximal with respect to containing no nonzero $R \# H$ submodule. We can now consider the chain of $R$-submodules

$$
M \supseteq \widehat{A}=\widehat{A}_{(1)} \supseteq \widehat{A}_{(2)} \supseteq \cdots \supseteq \widehat{A}_{(N-1)} \supseteq \widehat{A}_{(N)}=0 .
$$

Now suppose that $M$ is irreducible (resp. uniform) as a left $R \# H$-module. Since $B_{(N)} \neq 0$, for any $R$-submodule $B$ properly containing $\widehat{A}$, we see that the factor $R$-module $M / \widehat{A}$ is irreducible (resp. uniform). If $1 \leq i \leq N-1$, then we can consider the maps

$$
\varphi_{i}: \widehat{A}_{(i)} \rightarrow M / \widehat{A}
$$


defined as $\varphi_{i}(a)=h_{i+1} a+\widehat{A}$, for all $a \in \widehat{A}_{(i)}$. By (ii) there exist $\sigma, \tau \in G$ such that $\Delta\left(h_{i+1}\right)-\sigma \otimes h_{i+1}-h_{i+1} \otimes \tau \in H_{i} \otimes H_{i}$. Hence if $r \in R$ and $a \in \widehat{A}_{(i)}$, then since $H_{i} a \subseteq \widehat{A}$, we have

$$
\begin{aligned}
\varphi_{i}(r a) & =h_{i+1}(r a)+\widehat{A}=\left(h_{i+1} r\right) a+\widehat{A}=\sigma(r) h_{i+1} a+\left(h_{i+1} \cdot r\right) \tau a+\widehat{A} \\
& =\sigma(r) h_{i+1} a+\widehat{A}=\sigma(r) \varphi_{i}(a) .
\end{aligned}
$$

It is easy to see that $\operatorname{ker} \varphi_{i}=\widehat{A}_{(i+1)}$. Therefore each $\varphi_{i}$ induces an embedding of the lattice of $R$-submodules of $\widehat{A}_{(i)} / \widehat{A}_{(i+1)}$ into the lattice of $R$-submodules of $M / \widehat{A}$. In our situation the $R$-module $M / \widehat{A}$ is irreducible, so each $\widehat{A}_{(i)} / \widehat{A}_{(i+1)}$ is either the zero module or irreducible (resp. uniform) as an $R$-module. Therefore $M$ has a finite length (resp. finite Goldie rank), not exceeding $N$, as an $R$-module.

Let $Q=Q(R)$ be the symmetric Martindale quotient ring of $R$. From the result of Montgomery (see [M2, Corollary 3.5]) it follows that when $H$ is pointed, the $H$-action on $R$ can be extended to an $H$-action on $Q$. Moreover, it is easy to see that if $H$ acts finitely on $R$, then every essential ideal of $R$ contains an $H$-stable ideal which is also essential in $R$ (see [GH, Lemma 9]). As a consequence of some basic properties of $Q$, we obtain the following.

Lemma 6. Let $H$ be a pointed Hopf algebra and let $R$ be a semiprime left $H$-module algebra such that $R^{H}$ is reduced and $L^{H} \neq 0$ for every nonzero $H$-stable left ideal $L$ of $R$. Suppose $R^{H}$ satisfies a multilinear identity of degree $d$ and $H$ acts on $R$ finitely of dimension $N$. Then

(1) $L^{H} \neq 0$, for every nonzero $H$-stable left ideal $L$ of $Q$.

(2) $Z\left(R^{H}\right) \subseteq Z\left(Q^{H}\right)$.

(3) $Q^{H}$ is reduced and satisfies the same multilinear identity as $R^{H}$.

(4) $H$ acts finitely of dimension $N$ on $Q$.

(5) If $R^{H}$ is almost central in $R$, then $Q^{H}$ is almost cental in $Q$.

(6) If in addition $R^{H} \subseteq Z$, then $Q^{H} \subseteq C$.

Proof. For (1), if $L$ is a nonzero $H$-stable left ideal of $Q$, then $\widehat{L}=L \cap R$ is a nonzero $H$-stable left ideal of $R$. By assumption $\widehat{L}^{H} \neq 0$, so $L^{H} \neq 0$.

Before proving (2), notice that if $I$ is an $H$-stable essential ideal of $R$, then l. $\operatorname{ann}_{R}\left(I^{H} \cap Z\left(R^{H}\right)\right)=0$. Indeed, it is clear that $L=1 . \operatorname{ann}_{R}\left(I^{H} \cap Z\left(R^{H}\right)\right)$ is an $H$-stable left ideal of $R$. If $L \neq 0$, then $0 \neq I \cdot L \subseteq I \cap L$ and since $R^{H}$ is reduced, we obtain that $(I \cap L)^{H}$ is a two-sided ideal of $R^{H}$. By assumption $R^{H}$ satisfies a PI, so $0 \neq Z\left((I \cap L)^{H}\right) \subseteq Z\left(R^{H}\right)$. Thus one can choose a nonzero element $c \in(I \cap L)^{H} \cap Z\left(R^{H}\right)$. But then $c^{2} \in L \cdot\left(I^{H} \cap Z(R)\right)=0$, which is impossible, since $R^{H}$ is reduced. This also implies that $\operatorname{lann}_{Q}\left(I^{H} \cap Z\left(R^{H}\right)\right)=0$. By using an easy induction argument, we obtain that for any $d \geq 1$,

$$
\text { l. } \operatorname{ann}_{Q}\left(\left(I^{H} \cap Z\left(R^{H}\right)\right)^{d}\right)=0 .
$$

This immediately implies that $Z\left(R^{H}\right) \subseteq Z\left(Q^{H}\right)$. To see this, take a nonzero $q \in Q^{H}$ and an essential $H$-stable ideal $J$ of $R$ satisfying $J q \subseteq R$ and $q J \subseteq R$. Then, for any $x \in J^{H}$ and $c \in Z\left(R^{H}\right)$, we have $q x \in R^{H}$ and

$$
0=[q x, c]=[q, c] x+q[x, c]=[q, c] x .
$$

Hence $[q, c] J^{H}=0$ and by (2.1), $[q, c]=0$. Consequently, $Z\left(R^{H}\right) \subseteq Z\left(Q^{H}\right)$. This ends the proof of (2). 
For the first part of (3), take $q \in Q^{H}$ such that $q^{2}=0$, and $I$ an essential $H$-stable ideal of $R$ satisfying $q I \cup I q \subseteq R$. Then $\left(I^{H} \cap Z\left(R^{H}\right)\right) q \subseteq R$ and using (2) we obtain $\left(\left(I^{H} \cap Z\left(R^{H}\right)\right) q\right)^{2}=q^{2}\left(I^{H} \cap Z\left(R^{H}\right)\right)^{2}=0$. Since $\bar{R}^{H}$ is reduced, $\left(I^{H} \cap Z\left(R^{H}\right)\right) q=0$ and (2.1) forces that $q=0$. Therefore $Q^{H}$ is reduced.

Now let $f\left(x_{1}, x_{2}, \ldots, x_{d}\right)=\sum_{\sigma \in S_{d}} a_{\sigma} x_{\sigma(1)} x_{\sigma(2)} \ldots x_{\sigma(d)}$ be a multilinear polynomial such that the identity $f\left(x_{1}, x_{2}, \ldots, x_{d}\right)=0$ is satisfied by $R^{H}$. Take $q_{1}, q_{2}, \ldots, q_{d} \in Q^{H}$ and an $H$-stable essential ideal $I$ of $R$ such that $q_{j} I \subseteq R$ for $j=1,2, \ldots, d$. Then for all $c_{1}, c_{2}, \ldots, c_{d} \in I^{H} \cap Z\left(R^{H}\right)$ we have $c_{i} q_{i} \in R^{\bar{H}}$, so by using $(2)$,

$$
0=f\left(c_{1} q_{1}, c_{2} q_{2}, \ldots, c_{d} q_{d}\right)=f\left(q_{1}, q_{2}, \ldots, q_{d}\right) c_{1} c_{2} \ldots c_{d} .
$$

This means that $f\left(q_{1}, q_{2}, \ldots, q_{d}\right) \in 1 \cdot \operatorname{ann}_{Q}\left(\left(I^{H} \cap Z\right)^{d}\right)=0$. Thus the identity

$$
f\left(x_{1}, x_{2}, \ldots, x_{d}\right)=0
$$

is satisfied also by $Q^{H}$. This proves (3).

For (4), let $\widehat{\pi}: H \rightarrow \operatorname{End}_{K}(Q)$ be the natural $K$-algebra homomorphism, corresponding to the action of $H$ on $Q$. We need to show that $\operatorname{ker} \pi=\operatorname{ker} \widehat{\pi}$. The inclusion $\operatorname{ker} \pi \supseteq \operatorname{ker} \widehat{\pi}$ is clear. Suppose $h \in \operatorname{ker} \pi$. Take $q \in Q$ and $I$ an essential $H$-stable ideal of $R$ such that $q I \subseteq R$. Since $\widehat{\pi}(h)$ is an $R^{H}$-bimodule map, we obtain that

$$
\widehat{\pi}(h)(q) a=\widehat{\pi}(h)(q a)=\pi(h)(q a)=0
$$

for any $a \in I^{H}$. Hence $\widehat{\pi}(h)(q) \in 1 \cdot \operatorname{ann}_{Q}\left(I^{H}\right) \subseteq \operatorname{l.ann}_{Q}\left(I^{H} \cap Z\left(R^{H}\right)\right)=0$. Thus $h \in \operatorname{ker} \widehat{\pi}$ and consequently ker $\widehat{\pi}=\operatorname{ker} \pi$. Thus $\operatorname{dim}_{K} \widehat{\pi}(H)=\operatorname{dim}_{K} \pi(H)$.

For (5), if $L$ is a nonzero $H$-stable left ideal of $Q$, then $\widehat{L}=L \cap R$ is a nonzero $H$-stable left ideal of $R$. Since $Z(R) \subseteq C$ and $\widehat{L}^{H} \cap Z(R) \neq 0$, we obtain that $L^{H} \cap C \neq 0$. Thus $Q^{H}$ is almost central in $Q$.

For (6), take $q \in Q^{H}$ and an $H$-stable essential ideal $I$ of $R$ such that $q I \subseteq R$. If $c \in I^{H}$, then $q c \in R^{H} \subseteq Z$ and hence

$$
(q r-r q) c=(q r) c-r(q c)=(q c) r-(q c) r=0,
$$

for any $r \in R$. Thus $r q-q r \in \operatorname{l.ann}_{Q}\left(I^{H} \cap Z\left(R^{H}\right)\right)=0$. Therefore $q$ centralizes $R$, so $q \in C$.

We are now ready to prove the first main result of the paper.

Proof of Theorem 3. By Lemma 6, all assumptions on $R$ can be lifted to $Q$. Let $h_{1}, h_{2}, \ldots, h_{N} \in H$ be such that $\left\{\pi\left(h_{1}\right), \pi\left(h_{2}\right), \ldots, \pi\left(h_{N}\right)\right\}$ is a basis for $\pi(H) \subseteq$ $\operatorname{End}_{K}(Q)$. Notice that for any $q \in Q$ the left ideal $L=\sum_{i=1}^{N} Q\left(h_{i} \cdot q\right)$ is $H$-stable. By applying the remarks after Lemma 1, we see that any finitely generated (as a left $Q$-module) left ideal of $Q$ is contained in an $H$-stable finitely generated left ideal which is also injective as a $C^{H}$-module.

Let $M$ be a maximal ideal of $C^{H}$ and $\eta_{M}: Q \rightarrow Q_{M}$ be a natural ring homomorphism, where $Q_{M}$ is the localization of $Q$ at $S=C^{H} \backslash M$. By Lemma 1 it follows that $Q_{M}$ is semiprime and Lemma 6 shows that $\left(Q_{M}\right)^{H}=\left(Q^{H}\right)_{M}$ satisfies a multilinear identity of degree $d$. We claim that $\left(Q^{H}\right)_{M}$ is almost central in $Q_{M}$. Take a nonzero $H$-stable left ideal $T$ of $Q_{M}$ and choose a finitely generated $H$-stable left ideal $L$ of $Q$ such that $0 \neq \eta_{M}(L) \subseteq T$. Then $L$ is injective as a left $C^{H}$-module and by Lemma 1(3), $L^{H}$ is also injective as a left $C^{H}$-module. Since $C$ is injective over $C^{H}$, the intersection $L^{H} \cap C$ is injective as a $C^{H}$-module. By Lemma 1(2), there exist $x \in L^{H} \cap C$ and an idempotent $\widehat{e}_{x} \in C^{H}$ such that 
$\operatorname{ann}_{C^{H}}\left(L^{H} \cap C\right)=\operatorname{ann}_{C^{H}}(x)=\left(1-\widehat{e}_{x}\right) C^{H}$. We claim that $\left(1-\widehat{e}_{x}\right) L=0$. If not, then $\left(1-\widehat{e}_{x}\right) L$ is a nonzero $H$-stable left ideal of $Q$. Since $Q^{H}$ is almost central in $Q$, we can choose a nonzero $c \in\left(\left(1-\widehat{e}_{x}\right) L\right)^{H} \cap C$. Then $c \in L^{H} \cap C$ and $c=\left(1-\widehat{e}_{x}\right) c \in\left(1-\widehat{e}_{x}\right) C^{H}=\operatorname{ann}_{C^{H}}\left(L^{H} \cap C\right)$. Therefore $c^{2}=0$, which is impossible because $C$ is reduced. This proves the claim. Since $\eta_{M}(L) \neq 0,1-\widehat{e}_{x} \in M$. Hence $\operatorname{ann}_{C^{H}}(x) \subseteq M$ and thus $0 \neq \eta_{M}(x) \in T^{H} \cap C_{M}$. Therefore $\left(Q_{M}\right)^{H}$ is almost central in $Q_{M}$.

On the other hand by Lemma 1(5), $\left(Q_{M}\right)^{H} \cap C_{M}$ is a field, so $Q_{M}$ does not contain proper $H$-stable left ideals. Thus $Q_{M}$ is an irreducible left $Q_{M} \# H$-module. By Lemma 5. $Q_{M}$ has finite length as a left $Q_{M}$-module, so in particular $Q_{M}$ has finite left Goldie rank. We are now in a position to apply Theorem 2. It asserts that $\left(Q_{M}\right)^{H}$ is a division ring and $\left[Q_{M}:\left(Q_{M}\right)^{H}\right]_{r}=n \leq N$. If we let $A_{M}$ denote the annihilator ideal $\left\{w \in Q_{M} \# H \mid w Q_{M}=0\right\}$, then $Q_{M} \# H / A_{M} \simeq$ $M_{n}\left(\left(Q_{M}\right)^{H}\right)$. The division algebra $\left(Q_{M}\right)^{H}$ satisfies a polynomial identity of degree $d$, so $M_{n}\left(\left(Q_{M}\right)^{H}\right)$ satisfies the standard polynomial identity $s_{d n}$ of degree $d n \leq d N$. Since $Q_{M}$ is semiprime we have an embedding $Q_{M} \hookrightarrow Q_{M} \# H / A_{M}$. Thus for any maximal ideal $M$ of $C^{H}$, the localization $Q_{M}$ satisfies the standard polynomial identity $s_{d N}$. The fact that $C^{H}$ is von Neumann regular implies immediately the existence of an embedding $Q \hookrightarrow \prod_{M} Q_{M}$, where the product is taken over all maximal ideals of $C^{H}$. Therefore $Q$ satisfies $s_{d N}$.

If we additionally assume that $R^{H} \subseteq Z$, then by Lemma 6(6), $Q^{H} \subseteq C$. Thus for a given maximal ideal $M$ of $C^{H}, Q_{M}$ is a semisimple finite-dimensional algebra containing a central subfield $\left(C_{M}\right)^{H}$ such that $\operatorname{dim}_{\left(C_{M}\right)^{H}} Q_{M} \leq N$. Therefore, the Amitsur-Levitzki Theorem asserts that $Q_{M}$ satisfies the standard polynomial identity of degree $2[\sqrt{N}]$. As a result, if the invariants $R^{H}$ are central in $R$, then $R$ satisfies $s_{2[\sqrt{N}]}$, thereby concluding the proof.

Proof of Theorem 4. Let us first consider the special case where $R^{H}$ is a domain. Then, by Posner's Theorem, $R^{H}$ is a Goldie ring. Furthermore, if we put $T=$ $Z\left(R^{H}\right) \backslash\{0\}$, then the localization $T^{-1} R^{H}$ is a division algebra with center $\mathcal{Z}=$ $T^{-1} Z\left(R^{H}\right)$ and $\operatorname{dim}_{\mathcal{Z}} T^{-1} R^{H} \leq\left(\frac{d}{2}\right)^{2}$. It is easy to see that every nonzero element $z \in Z\left(R^{H}\right)$ is regular in $R$. In fact, since $R$ is reduced, $J=1 \cdot \operatorname{ann}_{R}(z)=$ r.ann $R(z)$ is a two-sided $H$-stable ideal of $R$. If $J$ is nonzero, then $Z\left(J^{H}\right) \neq 0$ (because $R^{H}$ satisfies a PI), and clearly $Z\left(J^{H}\right) \subseteq Z\left(R^{H}\right)$. But $Z\left(J^{H}\right) z=0$, and this contradicts our assumption that $R^{H}$ is a domain. We claim that the subset $T$ satisfies the left Ore condition in $R$. To see this, note that by Lemma 5, $R$ has a finite left Goldie rank. Furthermore $R$, as a reduced ring, certainly has zero singular ideal. Thus $R$ is left Goldie. Now it is enough to show that any essential left ideal of $R$ intersects $T$ nontrivially. Since the group $G=G(H)$ of group-like elements acts finitely, we need only consider essential left ideals which are $G$-stable. Let $L$ be a $G$-stable essential left ideal of $R$ and, using the notation in Lemma 5 , let $L_{(j)}=\left\{x \in L \mid h_{1} \cdot x, \ldots, h_{j} \cdot x \in L\right\}$. We will show by induction that $L_{(j)}$ is essential for all $j \geq 1$. To see this, let $I$ be any nonzero left ideal of $R$. Given $0 \neq a \in L_{(j-1)} \cap I$, the left ideal $E=\bigcap_{i \leq j}\left\{r \in R \mid r\left(h_{i} \cdot a\right) \in L\right\}$ is essential. Since $R$ is nonsingular, we can choose $r \in \bigcap_{\sigma \in G} E^{\sigma}$ with $r a \neq 0$. Then

$$
h_{j} \cdot(r a)=\sigma(r)\left(h_{j} \cdot a\right)+\left(h_{j} \cdot r\right) \tau(a)+\sum\left(h_{j 1} \cdot r\right)\left(h_{j 2} \cdot a\right) \in L .
$$


Thus $0 \neq r a \in L_{(j)}$, so $L_{(j)}$ is essential. In particular $\widehat{L}=L_{(N)}$ is nonzero and $H$-stable. By assumption $\widehat{L}^{H}$ is a nonzero left ideal of $R^{H}$. But $R^{H}$ is a PI domain, so any nonzero left ideal intersects $T$ nontrivially. This proves the claim.

Notice that $T^{-1} R$ is in a natural way a left $H$-module algebra and $\left(T^{-1} R\right)^{H}$ $=T^{-1} R^{H}$ is a division algebra satisfying a polynomial identity of degree $d$. It is also clear that $T^{-1} R$ has no proper left $H$-stable ideals, so $T^{-1} R$ becomes an irreducible left $\left(T^{-1} R\right) \# H$-module. Applying the same argument as in the proof of our previous theorem, we obtain that $T^{-1} R$ satisfies $s_{d N}$. Therefore $R$ also satisfies the standard identity $s_{d N}$.

For the general case, since $R$ is reduced, the symmetric Martindale quotient ring $Q$ is also reduced. Similarly, as in Theorem 3, let us consider a maximal ideal $M$ of $C^{H}$ and the canonical map $\eta_{M}: Q \rightarrow Q_{M}$. We claim that $\left(Q^{H}\right)_{M}$ is a domain. To this end, let $a, b \in Q^{H}$ be such that $a b=0$, and let $e_{a}, e_{b} \in C^{H}$ be idempotents such that $\operatorname{ann}_{Q}(Q a Q)=\left(1-e_{a}\right) Q$ and $\operatorname{ann}_{Q}(Q b Q)=\left(1-e_{b}\right) Q$. Since $Q$ is reduced, $\operatorname{lann}_{Q}(a)=\operatorname{r}_{\operatorname{ann}}(a)=\operatorname{ann}_{Q}(Q a Q)$. Thus r.ann $(a)=\left(1-e_{a}\right) Q$. Hence $e_{a} b=0$. On the other hand $\operatorname{lann}_{Q}(b)=\left(1-e_{b}\right) Q$, so there exists $x \in Q$ satisfying $e_{a}=\left(1-e_{b}\right) x$. Now it is clear that $e_{a} e_{b}=0$, and thus either $e_{a} \in M$ or $e_{b} \in M$. This immediately implies that either $\eta_{M}(a)=0$ or $\eta_{M}(b)=0$. Therefore $\left(Q^{H}\right)_{M}$ is a domain, as claimed. Notice that the ring $Q_{M}$ is reduced. Indeed, if $q \in Q$ and $c \in C^{H} \backslash M$ are such that $c q^{2}=0$, then $(c q)^{2}=0$ and $c q=0$, since $Q$ is reduced. Consequently $\eta_{M}(q)=0$. As a result $Q_{M}$ is a reduced left $H$-module algebra and its subalgebra of invariants $\left(Q_{M}\right)^{H}=\left(Q^{H}\right)_{M}$ is a domain satisfying a polynomial identity of degree $d$. By the previous paragraph $Q_{M}$ satisfies $s_{d N}$. Since this holds for any maximal ideal $M$ of $C^{H}$, the $\operatorname{ring} Q$ satisfies $s_{d N}$.

We close the paper with a remark concerning actions on reduced algebras. We see that the result of Kharchenko for group actions (mentioned in the introduction) is a direct consequence of his fundamental result on the existence of fixed elements and Theorem 4. Moreover, Beidar and Grzeszczuk proved in [BeG] an analogous result on the existence of nontrivial constants for actions of Lie algebras. Finally, Theorem 4 now provides us with a common proof of the following.

Corollary 7. Let $R$ be a reduced algebra. Then

(1) (cf. [K2]) if $R$ is acted on by a finite group $G$ and $R^{G}$ satisfies a PI of degree $d$, then $R$ satisfies a PI of degree $d|G|$.

(2) If $R$ is acted finitely on by a finite-dimensional Lie algebra $L$ and $R^{L}$ satisfies a PI of degree $d$, then $R$ satisfies a PI of degree $d N$, where $N$ is the dimension of the action.

\section{REFERENCES}

[AS] N. Andruskiewitsch, H.J. Schneider, Pointed Hopf Algebras, New directions in Hopf Algebras. Math. Sci. Res. Inst. Publ., 1-68, 43, Cambridge Univ. Press, Cambridge, 2002, 634-654. MR1913436 (2003e:16043)

[BaL] Y. Bahturin, V. Linchenko, Identities of algebras with actions of Hopf algebras, J. Algebra 202 (1998), 634-654. MR.1617671 (99d:16040)

[BaZ] Y. Bahturin, M. Zaicev, Identities of graded algebras, J. Algebra 205 (1998), 1-12. MR.1631298 (99f:17034)

[BeG] K.I. Beidar, P. Grzeszczuk, Actions of Lie algebras on rings without nilpotent elements, Algebra Colloq. 2(2) (1995), 105-116. MR1329141 (96f:16043)

[BeT] K.I. Beidar, B. Torrecillas, On actions of Hopf algebras with cocommutative coradical, J. Pure and Applied Algebra, 161 (2001), 13-30. MR1834076 (2002f:16080) 
[BC] J. Bergen, M. Cohen, Actions of commutative Hopf algebras, Bull. London Math. Soc., 18 (1986), 159-164. MR0818820 (87e:16052)

[BCF] J. Bergen, M. Cohen, D. Fischman, Irreducible actions and faithful actions of Hopf algebras, Israel J. Math., 72 (1990), 5-18. MR1098978 (92g:16044)

[BG] J. Bergen, P. Grzeszczuk, Invariants of Lie superalgebras acting on associative rings, Israel J. Math. 94 (1996), 403-428. MR1394584 (97g:16046)

[C] M. Cohen, Quantum commutativity and central invariants, Advances in Hopf algebras, Lecture Notes in Pure and Appl. Math., 158, Dekker, New York, 1994, 25-38. MR1289420 (95h:16050)

[CW] M. Cohen, S. Westreich, Central invariants of H-module algebras, Comm. Algebra, 21(8) (1993), 2859-2883. MR1222747 (94d:16034)

[GH] P. Grzeszczuk, M. Hryniewicka, Polynomial identities of algebras with actions of pointed Hopf algebras, J. Algebra, 278 (2004), 684-703. MR2071660(2005d:16037)

[K1] V. K. Kharchenko, Generalized identities with automorphisms, Algebra i Logika 14 (1975), 215-237 (English translation 1976, 132-148). MR0399153(53:3004)

[K2] V. K. Kharchenko, Fixed elements under a finite group acting on a semiprime ring, Algebra i Logika 14 (1975), 328-344 (English translation 1976, 409-417). MR0429998 (55:3006)

[K3] V. K. Kharchenko, J. Keller, S. Rodrigues-Romo, Prime rings with PI rings of constants, Israel J. Math. 96 (1996), 357-377. MR1433695 (97k:16052)

[M1] S. Montgomery, Hopf algebras and their actions on rings, CBMS Regional Conference Series in Mathematics 82, Amer. Math. Soc., Providence, R.I., 1993. MR1243637|(94i:16019)

[M2] S. Montgomery, Bi-invertible actions of Hopf algebras, Israel J. Math., 83 (1993), no. 1-2, 45-71. MR 1239716 (94g:16047)

Faculty of Computer Science, Technical University of BiaŁystok, Wiejska 45A, 15351 BiaŁystok, Poland

E-mail address: piotrgr@pb.bialystok.pl

Institute of Mathematics, University of BiaŁystok, Akademicka 2, 15-267 Bialystok, POLAND

E-mail address: margitt@math.uwb.edu.pl 